\title{
Spontaneous Pneumothorax Complicating Hemodialysis
}

\author{
SUNIL KUMAR ${ }^{1}$, SK DIWAN ${ }^{1}$, AP SINGH ${ }^{1}$, S. KOPPATIWAR ${ }^{1}$
}

Pneumothorax is an uncommon but a well-recognized complication of hemodialysis catheter or central line insertion in internal jugular vein or subclavian vein. Subclavian catheterization is more likely than internal jugular catheterization to be complicated by pneumothorax and hemothorax ${ }^{1}$. Data regarding spontenous pneumothorax during hemodialysis are not available, and in our opinion this is probably the first case report. A 58year-old tribal man was admitted in medicine department for complain of weakness, puffiness of face, oedema in lower limb. His investigation revealed total leucocyte count

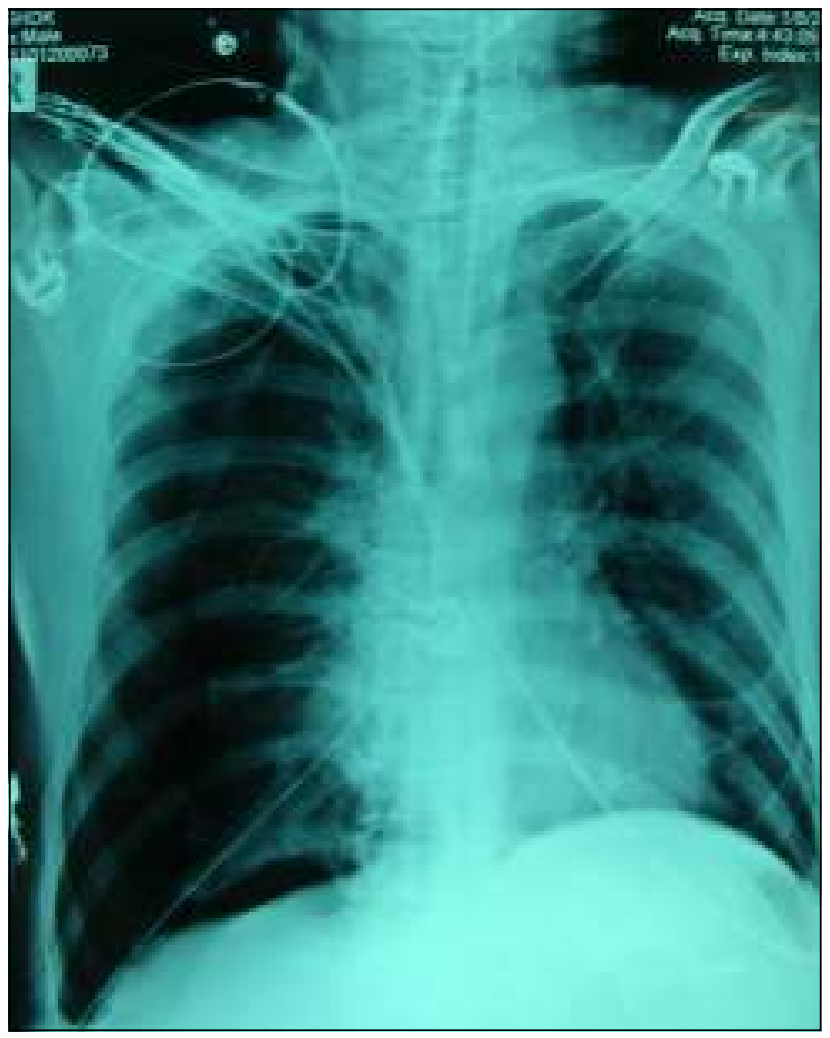

Fig.-1
$16000 / \mathrm{cmm}$, neutrophil $80 \%, \mathrm{Hb} 6 \mathrm{gm} / \mathrm{dl}$, serum creatinine $6.5 \mathrm{mg} / \mathrm{dl}$, blood urea $138 \mathrm{mg} / \mathrm{dl}$, and serum potassium 5.2 $\mathrm{mEq} / \mathrm{l}$. His fasting blood sugar was $96 \mathrm{mg} / \mathrm{dl}$ and postprandial glucose was $138 \mathrm{mg} / \mathrm{dl}$. Due to deteriorated renal function he underwent hemodialysis with a double lumen hemodialysis catheter in right internal jugular vein by seldinger technique under full aseptic precaution in the dialysis unit of the medicine department. He was doing well. On day $18^{\text {th }}$ during the process of hemodialysis he complained of acute breathlessness with diaphoresis. Blood sugar and blood pressure were checked which were normal. On chest examination breath sound was absent on right side. Chest $\mathrm{x}-$ ray was obtained which revealed pneumothorax (fig). Water sealed intercostal tube was inserted, pneumothorax improved as well as his breathlessness. We searched the literature, textbook, pubmed, medlar, there was no any case report or article regarding this and its mechanism. Probable hypothesis may be secondary to pulmonary embolisation either due to central venous catheter infection ${ }^{2}$ or sometimes hemodialysis machine itself which doesnot censor micro air emboli. The pneumothorax was thought to be due to rupture of the subpleural lesions. Any of the above explanations or some other unknown mechanisms may explain its association we may not accept or reject any, and further research is needed.

\section{Conflict of interest: None}

\section{References:}

1. David C M, Michael K G. Preventing Complications of Central Venous Catheterization. N Engl J Med 2003; 348:1123-33.

2. C C Sheu, J J Hwang, J R Tsai, T H Wang, I W Chong, M S Huang. spontaneous pneumothorax as a complication of septic pulmonary embolism in i.v. drug user: a case report. Kaohsiung J Med Sci 2006; 22:89-93

1. Department of Medicine, Jawahar Lal Nehru Medical College, DMIMS, Sawangi, wardha.

Correspondence : Dr. Sunil Kumar, Associate professor, Department of medicine. DMIMS, Sawangi, wardha; E-mail sunilkumarmed@gmail.com 